\title{
Nefritis lúpica: experiencia con dosis reducidas de glucocorticoides en una unidad de enfermedades autoinmunes sistémicas
}

\author{
Lupus nephritis: low-dose glucocorticoids in a systemic autoimmune diseases unit \\ Nefritis lúpica: experiência con doses reduzidas de glicocorticoides em uma unidade \\ de doenças autoinmunes sistêmicas
}

\author{
Adriana Carlomagno ${ }^{1}$, Gonzalo Silveira¹, Álvaro Danza², Ana Carina Pizzarossa', \\ Martín Yandián ${ }^{3}$, Federico Yandián ${ }^{4}$, Martín Rebella ${ }^{5}$
}

\section{Resumen}

Introducción: las recomendaciones actuales del tratamiento de la nefritis lúpica (NL) apuntan a dosis de glucocorticoides más bajas para lograr el control de la enfermedad y evitar el daño acumulado.

Objetivo: conocer y comparar la respuesta al tratamiento de pacientes con NL proliferativa en su etapa de inducción con dos pautas de tratamiento con prednisona (PDN): dosis iniciales reducidas $\leq 30 \mathrm{mg} / \mathrm{d}$ y dosis iniciales estándar $>30 \mathrm{mg} / \mathrm{d}$.

Método: se compararon variables clínicas, analíticas y terapéuticas de pacientes con NL proliferativa categorizados en dos grupos según la dosis inicial de prednisona (PDNi) estándar o reducida.

Resultados: se estudiaron 21 pacientes con NL proliferativa ( $n=12$ PDNi reducida vs. $n=9$ PDNi estándar). No hubo diferencias significativas en las variables clínicas y analíticas. Se observó una diferencia estadísticamente significativa en el número de pulsos de metilprednisolona ( $5 \pm 2,95 \mathrm{PDNi} \leq 30 \mathrm{mg} / \mathrm{d}$ vs 2,33 $\pm 2,91 \mathrm{PDNi}>30 \mathrm{mg} / \mathrm{d}, \mathrm{p}=0,041)$ y en la dosis de prednisona acumulada a 6 meses (12,8 mg $\pm 4,9 \mathrm{PDNi} \leq 30 \mathrm{mg} / \mathrm{d}$ vs $30,0 \pm 13,1 \mathrm{mg} P D N i>30 \mathrm{mg} / \mathrm{d}, \mathrm{p}=0,008)$. No hubo diferencias significativas en la proporción de pacientes que alcanzaron la respuesta completa, en el tiempo hasta alcanzarla ni en los efectos adversos entre ambos grupos.

Conclusiones: el esquema terapéutico del grupo PDNi $\leq 30 \mathrm{mg} / \mathrm{d}$ se asoció a una menor dosis acumulada de prednisona y una respuesta al tratamiento comparable, lo que hace presumir menor daño acumulado relacionado al uso de glucocorticoides.

\footnotetext{
1. Internista. Asistente de Clínica Médica. Facultad de Medicina, Universidad de la República. Unidad de Enfermedades Autoinmunes Sistémicas de Médica Uruguaya Corporación de Asistencia Médica.

2. Profesor Agregado de Clínica Médica. Facultad de Medicina, Universidad de la República. Unidad de Enfermedades Autoinmunes Sistémicas de Médica Uruguaya Corporación de Asistencia Médica.

3. Profesor Adjunto de Clínica Médica. Facultad de Medicina, Universidad de la República. Unidad de Enfermedades Autoinmunes Sistémicas de Médica Uruguaya Corporación de Asistencia Médica.

4. Nefrólogo. Asistente del Centro de Nefrología del Hospital de Clínicas. Facultad de Medicina, Universidad de la República. Unidad de Enfermedades Autoinmunes Sistémicas de Médica Uruguaya Corporación de Asistencia Médica.

5. Profesor Adjunto de Clínica Médica. Facultad de Medicina, Universidad de la República. Montevideo, Uruguay. Coordinador Unidad de Enfermedades Autoinmunes Sistémicas de Médica Uruguaya Corporación de Asistencia Médica.

Correspondencia: Dr. Martín Rebella. Correo electrónico: mrebella76@gmail.com

Los autores declaran no presentar conflictos de intereses.

No se obtuvo financiación específica para este estudio.

Recibido: 22/4/2021

Aprobado: 14/7/2021

Attribution-NonCommercial 4.0 International (CC BY-NC 4.0)
} 
Palabras clave: Nefritis lúpica

Glucocorticoides

Prednisona

Key words: $\quad$ Lupus nephritis

Glucocorticoids

Prednisone

\section{Introducción}

La nefritis lúpica (NL) es la manifestación grave más frecuente del lupus eritematoso sistémico (LES), siendo las formas proliferativas las más frecuentes ${ }^{(1)}$. Afecta entre $35 \%-65 \%$ de los pacientes con LES dependiendo de la etnia, sexo y edad, entre otros factores ${ }^{(2,3)}$. Es más frecuente en la población afrodescendiente e hispa$n a^{(4,5)}$, quienes además presentan evoluciones más adversas y resultados a largo plazo menos favorables ${ }^{(6)}$. De acuerdo con el Registro Uruguayo de Glomerulopatías, la glomerulonefritis lúpica es la cuarta en incidencia, con un $12,2 \%$ de todas las biopsias analizadas, $y$ ocupa el segundo lugar en incidencia de glomerulopatías secundarias ${ }^{(7,8)}$.

La morbimortalidad del LES se ha modificado sustancialmente $^{(9)}$, encontrándose previamente relacionada a la actividad de la enfermedad y a los efectos adversos a corto plazo del tratamiento empleado para inducir la remisión. Actualmente está determinada fundamentalmente por el daño orgánico acumulado que depende de la actividad sostenida en el tiempo de la enfermedad, la aparición de brotes y los efectos adversos de la terapéutica empleada. Un factor clave está relacionado al uso de dosis altas y exposición prolongada a glucocorticoides, con los efectos adversos que ello determina: osteonecrosis, fracturas osteoporóticas, cataratas, diabetes, enfermedades cardiovasculares, y cambios físicos que impactan en la calidad de vida los pacientes, entre otros bien conocidos ${ }^{(10-13)}$.

El tratamiento de las manifestaciones severas del LES consiste en inducir la remisión de manera rápida, o lograr el menor nivel de actividad posible, y que esto se mantenga en el tiempo. El tratamiento estándar de las formas proliferativas de NL consiste en la administración de inmunosupresores asociados a glucocorticoides e hidroxicloroquina ${ }^{(14,15)}$. En el caso particular de la NL consiste en reducir o suprimir la proteinuria y normalizar o mejorar el sedimento urinario y el filtrado glomerular, evitando el deterioro de la función renal a largo plazo. Es evidente que esto debe hacerse con la menor carga de efectos adversos para el paciente y sin impactar sustancialmente en la calidad de vida.

Aun cuando la evidencia respecto a la efectividad y seguridad de dosis reducidas de glucocorticoides viene en aumento, sigue siendo habitual el uso de dosis altas de prednisona por vía oral, $1 \mathrm{mg} / \mathrm{kg} / \mathrm{d}$. Esta dosis ha resultado efectiva, pero con una alta carga de efectos adversos. Los estudios clínicos en los que se comparan dosis elevadas con esquemas reducidos, aunque escasos, apoyan el uso de dosis menores a este esquema clásico. Las recomendaciones actuales del tratamiento postulan dosis de glucocorticoides más bajas para lograr el control de la enfermedad y evitar el daño acumulado ${ }^{(16)}$. Se ha documentado que dosis de prednisona acumuladas mayores a $7,5 \mathrm{mg} / \mathrm{d}$ durante el primer año de la enfermedad aumentan significativamente el daño acumulado a 5 años ${ }^{(16-18)}$. A su vez, las dosis iniciales de prednisona predicen las dosis acumuladas a los 11 meses. Es decir, si el tratamiento se inicia con dosis altas de prednisona, las dosis acumuladas también serán elevadas, y esto ocurre con independencia del nivel de actividad de la enfermedad ${ }^{(19)}$.

Dosis medias diarias mantenidas de 6-12 mg/d determinan un incremento en el riesgo de daño orgánico, tal como lo demuestra el análisis de la Cohorte del Lupus-Hopkins ${ }^{(10)}$. A partir de $5 \mathrm{mg} / \mathrm{d}$, los glucocorticoides aumentan el riesgo de infecciones graves que requieren hospitalización ${ }^{(20-22)}$. Se ha evidenciado un aumento del riesgo de reactivación de tuberculosis latente e infecciones oportunistas, como neumonía por Pneumocystis jirovecii, a partir de dosis menores de $15 \mathrm{mg} / \mathrm{d}^{(23-25)}$ y también un aumento del riesgo de reactivación del virus varicela zoster a partir de dosis de 7,5-10 mg/d, incrementándose este riesgo a medida que aumenta la dosis y el tiempo de exposición ${ }^{(22)}$.

Por otro lado, existe evidencia creciente que avala el uso combinado de pulsos de metilprednisolona (MTP) en el tratamiento de inducción de la NL, denominado actualmente "tratamiento inicial", seguido de dosis de prednisona (PDN) no mayores a $30 \mathrm{mg} / \mathrm{d}^{(26)}$. Se ha comprobado que este esquema es igualmente efectivo y con una menor carga de efectos adversos que el clásico con 1 $\mathrm{mg} / \mathrm{kg} / \mathrm{d}$, y permite la reducción de la prednisona a no más de 5 a $7,5 \mathrm{mg} / \mathrm{d}$ a las $12-15$ semanas (3 meses), lográndose así menores dosis acumuladas de PDN.

El objetivo de este trabajo es conocer y comparar la respuesta al tratamiento de pacientes con NL proliferativa en la etapa de tratamiento inicial, de inducción a la remisión y mantenimiento, con dos pautas de tratamiento con prednisona (PDN): dosis iniciales reducidas $\leq 30$ $\mathrm{mg} / \mathrm{d}$ y dosis iniciales estándar $>30 \mathrm{mg} / \mathrm{d}$.

\section{Materiales y métodos}

Se realizó un estudio retrospectivo en la Unidad de Enfermedades Autoinmunes Sistémicas de Médica Uruguaya Corporación de Asistencia Médica (MUCAM). Se incluyeron pacientes con lupus eritematoso sistémico según criterios clasificatorios del ACR de $1997^{(27)}$ y que presentaran NL III/IV confirmada histológicamente mediante la clasificación ISN-RPS 2003, asistidos en el 
Tabla 1. Plan de tratamiento con glucocorticoides en nefritis lúpica (primer año de seguimiento).

\begin{tabular}{|c|c|c|}
\hline Primer mes & $\begin{array}{l}\text { PDN } 30 \text { mg/d (15 días) } \\
\text { PDN } 20 \text { mg/d (15 días) }\end{array}$ & \multirow{4}{*}{$\begin{array}{l}\text { Iniciar precozmente: } \\
\text { - Hidroxicloroquina (en ausencia de } \\
\text { contraindicaciones, excepcional) } \\
\text { - Calcio + vitamina D } \\
\text { - IECA } \\
\text { - Inducción con: CFM } 500 \text { mg cada } 15 \text { días, } \\
\text { total } 6 \text { a } 9 \text { pulsos o MMF } 3 \text { g/d por } 6 \text { meses. } \\
\text { Mantenimiento con MMF } 2 \text { g/d mínimo } 3 \text { años. } \\
\text { - Pulsos de MTP (no mayores a } 500 \text { mg/d por } \\
3 \text { días) al inicio, luego } 250 \mathrm{mg} \text { intravenosos } \\
\text { con cada pulso de CFM }\end{array}$} \\
\hline Segundo mes & $\begin{array}{l}\text { PDN } 15 \text { mg/d (15 días) } \\
\text { PDN } 10 \text { mg/d (15 días) }\end{array}$ & \\
\hline Tercer mes & PDN 7,5 mg/d (1 mes) & \\
\hline Cuarto mes en adelante & $\begin{array}{l}\text { PDN } 5 \mathrm{mg} / \mathrm{d} \text { (mínimo } 1 \mathrm{mes} \text { ) } \\
\text { Intentar suspender } \\
\text { Recordar llegar a } 5 \mathrm{mg} / \mathrm{d} \text { en } 12 \text { - } 16 \text { semanas }\end{array}$ & \\
\hline
\end{tabular}

PDN: prednisona; MTP: metilprednisolona; CFM: ciclofosfamida; MMF: micofenolato mofetilo; IECA: inhibidores de la enzima conversora de angiotensina.

período 2016-2019 ${ }^{(28)}$. Se compararon dos grupos de pacientes: una cohorte de pacientes histórica, que había sido tratada según pautas estándar de uso respecto a la cohorte que viene siendo seguida por nuestro equipo.

Se analizaron las siguientes variables: demográficas (sexo y edad), clínicas (tabaquismo, diabetes mellitus, hipertensión arterial), analíticas (filtrado glomerular estimado por fórmula CKD-EPI) ${ }^{(29)}$, títulos de fracción C3 del complemento, anticuerpos anti-DNA cualitativos (considerados de forma dicotómica: positivos o negativos), proteinuria de $24 \mathrm{~h}$, dosis de prednisona acumulada a 6 meses de tratamiento $(\mathrm{mg} / \mathrm{d})$ y respuesta a 3,6 y 12 meses de tratamiento. A los efectos de este estudio, se definió respuesta completa como la normalización del sedimento urinario junto con el descenso de la proteinuria a menos de $0,7 \mathrm{~g} / 24 \mathrm{~h}$ y valor de creatininemia dentro de $25 \%$ respecto al basal ${ }^{(30)}$. La actividad de la enfermedad se registró de acuerdo con el índice de actividad: Safety of Estrogens in Lupus erythematosus: National Assessment-SLE Disease Activity Index (SLEDAI-2K) ${ }^{(31)}$.

A los 6 meses y al año se registraron efectos adversos de modo dicotómico, presentes o ausentes. Los efectos adversos considerados fueron: infecciones, hipertensión arterial, cataratas, glaucoma, dislipidemia, hiperglicemia, osteoporosis, dermatológicos (acné, estrías), síndrome de Cushing.

La población de estudio se dividió en dos grupos según la dosis inicial de prednisona (PDNi) estándar (>30 $\mathrm{mg} / \mathrm{d})$ o reducida $(\leq 30 \mathrm{mg} / \mathrm{d})$. La categorización en grupos según las dosis iniciales de prednisona fue considerada teniendo en cuenta el esquema terapéutico del Hospital Universitario de Cruces-Bilbao ${ }^{(32)}$. Este protocolo consiste en la administración de pulsos intravenosos de MTP en dosis de 250-500 mg durante tres días consecutivos y posteriormente dosis de prednisona oral de no más de $30 \mathrm{mg} / \mathrm{d}$, con reducción a $5 \mathrm{mg} / \mathrm{d}$ en un plazo no mayor a las 12-16 semanas (tabla 1). A este tratamiento se agrega el "estándar" de inmunosupresores, micofeno- lato de mofetilo (MMF) o ciclofosfamida (CFM), hidroxicloroquina, calcio y vitamina $\mathrm{D}$ e inhibidores de la enzima conversora de angiotensina si corresponde.

Las variables cuantitativas se presentan con medidas de tendencia central y dispersión; media y desvío estándar y mediana y rango, según su distribución. La normalidad se probó mediante test de hipótesis (Shapiro-Wilk) y gráficos. Las variables cualitativas se presentan mediante frecuencias absoluta y relativa. Las comparaciones entre grupos se realizaron mediante test de $\mathrm{Chi}^{2} \mathrm{o}$ test de Fisher para los datos cualitativos y mediante test de $t$ de Student o de Wilcoxon, según la distribución, para los datos cuantitativos. La duración de la respuesta hasta la recaída se comprobó mediante curvas de sobrevida de Kaplan Meier, que se compararon mediante el test de log-rank. Se consideró un nivel de significación estadística menor a 0,05 . Los datos se procesaron en el programa informático SPSS.

El trabajo se desarrolló de acuerdo con las normas éticas establecidas en la Declaración de Helsinki de la Asociación Médica Mundial. Se obtuvo autorización del Comité de Ética institucional. Los datos fueron tratados con total confidencialidad, para lo cual se anonimizó la información de los participantes.

\section{Resultados}

\section{Variables demográficas, clínicas, analíticas}

Se incluyeron 21 casos de NL. No hubo diferencias estadísticamente significativas en las características demográficas, clínicas, analíticas e índice de actividad de la enfermedad al momento del diagnóstico de NL entre los grupos $\mathrm{PDNi} \leq 30 \mathrm{mg} / \mathrm{d}$ vs. $>30 \mathrm{mg} / \mathrm{d}(\mathrm{p}>0,05)$ (tabla 2$)$.

\section{Tratamientos}

Todos los pacientes estaban bajo tratamiento con hidroxicloroquina al diagnóstico de NL. Siete pacientes del grupo $\mathrm{PDNi}>30 \mathrm{mg} / \mathrm{d}$ y 12 pacientes del grupo PDNi 
Tabla 2. Características demográficas, clínicas, analíticas e índice de actividad al momento de la inclusión de los pacientes al estudio.

\begin{tabular}{|c|c|c|c|}
\hline$N=21$ & $P D N i \leq 30 \mathrm{mg} / \mathrm{d}(n=12)$ & $P D N i>30 \mathrm{mg} / \mathrm{d}(n=9)$ & Valor $p$ \\
\hline Edad (años) & $24,2 \pm 10,7$ & $30 \pm 12,4$ & 0,24 \\
\hline Sexo femenino & 11 & 6 & 0,36 \\
\hline Tabaquismo & 0 & 2 & \\
\hline DM & 0 & 0 & \\
\hline HTA & 1 & 3 & \\
\hline FG previo (ml/min) & $100,4 \pm 26$ & $76 \pm 39,3$ & 0,16 \\
\hline C3 (mg/dl) & $59 \pm 19,6$ & $59 \pm 28,5$ & 1 \\
\hline Proteinuria 24 h (g/día) & $1,2(0,6-3,9)$ & $1,4(0,6-3,5)$ & 0,93 \\
\hline Anti-DNA positivo & 10 & 6 & 0,17 \\
\hline SLEDAI-2K al inicio & $19(10-22)$ & $18(16-22)$ & 0,59 \\
\hline Nefritis tipo III & $6 / 12$ & $4 / 9$ & 0,51 \\
\hline Nefritis tipo IV & $6 / 12$ & $5 / 9$ & 0,51 \\
\hline
\end{tabular}

DM: diabetes mellitus; HTA: hipertensión arterial; FG: filtrado glomerular; C3: fracción C3 del complemento; Anti-DNA: anticuerpo anti-ADN.

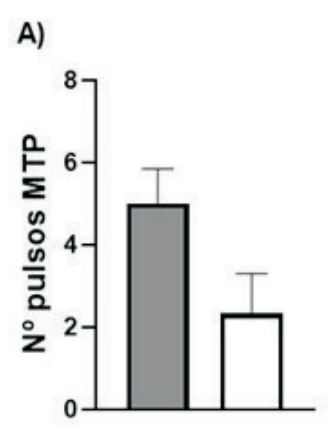

B)

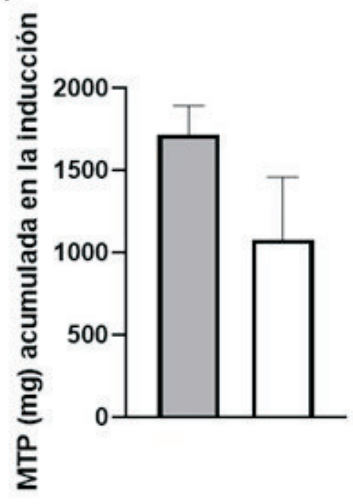

C)

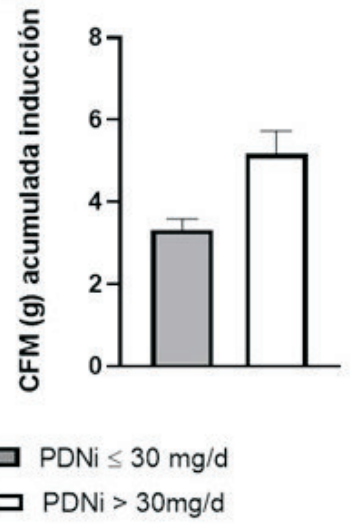

Figura 1. A) Media del número de bolos de MTP. La media fue de $5 \pm 2,95$ para el grupo PDNi $\leq 30 \mathrm{mg} / \mathrm{d}$ y 2,33 $\pm 2,91$ para el grupo PDNi $>30 \mathrm{mg} / \mathrm{d} ; \mathrm{p}=0,041$. B) Media de dosis de MTP acumulada en la inducción. La media fue de $1719 \pm 608$ para el grupo PDNi $\leq 30 \mathrm{mg} / \mathrm{d}$ y de $1.083 \pm 1.132$ para el grupo PDNi $>30 \mathrm{mg} / \mathrm{d}$. No se obtuvo diferencia estadísticamente significativa. C) Media de la dosis acumulada de CFM en la inducción. La media fue de $3,31 \pm 0,90$ para el grupo PDNi $\leq 30 \mathrm{mg} / \mathrm{d}$ y de 5,17 $\pm 1,48$ para el grupo PDNi $>30 \mathrm{mg} / \mathrm{d} ; \mathrm{p}=0,021$.

$\leq 30 \mathrm{mg} / \mathrm{d}$ recibieron calcio y vitamina $\mathrm{D}$. Cinco pacientes del grupo PDNi $\leq 30 \mathrm{mg} / \mathrm{d}$ y 8 pacientes del grupo $\mathrm{PDNi}>30 \mathrm{mg} / \mathrm{d}$ estaban bajo tratamiento con inhibidores de la enzima conversora de angiotensina (IECA); el resto no recibió este tratamiento porque se encontraba contraindicado, por hipotensión o por falta de adherencia. Se observaron diferencias estadísticamente significativas en el número de pulsos de MTP (figura 1A). No se observaros diferencias estadísticamente significativas en la medida de dosis acumuladas de MTP que reci- 
Tabla 3. Proporción de pacientes que alcanzaron la remisión completa a los 3, 6 y 12 meses de tratamiento.

\begin{tabular}{lccc}
\hline $\begin{array}{l}\text { Proporción de pacientes que } \\
\text { alcanzaron respuesta completa }\end{array}$ & $\begin{array}{c}P D N i \\
\leq 30 \mathrm{mg} / \mathrm{d}\end{array}$ & $\begin{array}{c}\mathrm{PDNi} \\
>30 \mathrm{mg} / \mathrm{d}\end{array}$ & \\
\hline 3 meses & $9 / 12$ & $3 / 6$ & $p>0,05$ \\
6 meses & $9 / 12$ & $6 / 8$ & \\
12 meses & $8 / 11$ & $7 / 8$ & \\
\hline
\end{tabular}

bieron los pacientes en el tratamiento de inducción (figura 1B). Hubo diferencias significativas en las dosis acumuladas de CFM en la inducción (figura 1C). Un paciente del grupo PDNi $\leq 30 \mathrm{mg} / \mathrm{d}$ y un paciente del grupo $\mathrm{PDNi}>30 \mathrm{mg} / \mathrm{d}$ recibió tratamiento de inducción con MMF, todos los demás recibieron tratamiento de inducción con CFM.

Se evidenció una diferencia estadísticamente significativa en la dosis de prednisona acumulada a 6 meses, siendo menor para el grupo con $\mathrm{PDNi} \leq 30 \mathrm{mg} / \mathrm{d}$ (figura 2). Con una media de dosis diaria acumulada de $12,8 \pm$ 4,9 mg/d; con un mínimo de $2,7 \mathrm{mg} / \mathrm{d}$ y máximo de 18,4 $\mathrm{mg} / \mathrm{d}$ para el grupo $\mathrm{PDNi} \leq 30 \mathrm{mg} / \mathrm{d}$. Para el grupo PDNi $>30 \mathrm{mg} / \mathrm{d}$ la media de dosis diaria acumulada fue de 30,0 $\pm 13,1 \mathrm{mg} / \mathrm{d}$, un mínimo de $18,6 \mathrm{mg} / \mathrm{d}$ y máximo de 59,4 $\mathrm{mg} / \mathrm{d} ; \mathrm{p}<0,01$.

\section{Respuesta terapéutica y efectos adversos}

No se observaron diferencias significativas en la proporción de pacientes que alcanzaron respuesta completa a los 3, 6, y 12 meses de tratamiento ( $\mathrm{p}>0,05)$ (tabla 3$)$, ni tampoco se evidenciaron diferencias significativas en el tiempo hasta alcanzar la respuesta completa (figura 3).

No se registraron diferencias significativas en los efectos adversos en ambos grupos en el período de observación del estudio.

\section{Discusión}

En nuestro trabajo observamos que no existen diferencias significativas en la proporción de pacientes que alcanza respuesta completa entre el grupo que inicia tratamiento con dosis reducidas de PDN respecto al grupo que recibió los clásicos esquemas de dosis elevadas. Tampoco se observaron diferencias en el tiempo de respuesta, habida cuenta que un aspecto crítico en el tratamiento de la NL no solo consiste en inducir la remisión, sino en hacerlo en el menor tiempo posible. Se trata de una observación interesante a la luz de que el grupo de pacientes considerados en uno y otro esquema tienen un comportamiento clínico e histopatológico similar.
En esa línea, se muestra que un tratamiento pautado y sistemático es importante en enfermedades de difícil manejo. No es menor la observación que el grupo de dosis reducidas de glucocorticoides se acompañe de una menor dosis acumulada de ciclofosfamida y un amplio uso de hidroxicloroquina. Es posible que en este aspecto exista sesgo puesto que los dos grupos de pacientes comparados fueron asistidos por equipos diferentes, con un protocolo muy estricto en nuestra cohorte. En el mismo sentido, no es llamativo que el grupo de dosis menores de PDN todos los pacientes recibieran calcio y vitamina $\mathrm{D}$, apoyando todo esto la importancia de la protocolización de los tratamientos.

Es ampliamente conocido que el uso prolongado de glucocorticoides se asocia a daño orgánico permanente ${ }^{(33-36)}$, sobre todo en aquellos pacientes expuestos a dosis mantenidas por encima de $20 \mathrm{mg} / \mathrm{d}^{(37,38)}$. Nuestro grupo ha comprobado, en consonancia con otros, que el daño a largo plazo está particularmente relacionado con las dosis iniciales, independientemente de los niveles de actividad considerados al inicio del cuadro clínico ${ }^{(18,19)}$. Teniendo en cuenta el perfil de riesgo asociado a los glucocorticoides, ha surgido la necesidad de desarrollar nuevos esquemas de dosificación que permitan, al mismo tiempo, inducir la remisión de la enfermedad y disminuir el riesgo de desarrollar daño orgánico permanente. Este ha sido el punto de partida para diversos estudios clínicos y farmacológicos.

Los glucocorticoides tienen dos vías de acción, una vía genómica y otra no genómica ${ }^{(39)}$, con un perfil diferente en cuanto a sus mecanismos de activación y de seguridad $^{(40)}$. La vía genómica se activa a partir de dosis bajas de PDN o equivalente, menores de 7,5 mg/d, y expresa su efecto antiinflamatorio en forma dosis dependiente, alcanzando una saturación cercana al 100\% con dosis de $30-40 \mathrm{mg} / \mathrm{d}$. Con dosis de prednisona superiores a $30 \mathrm{mg} / \mathrm{d}$ se incrementa el riesgo de efectos adversos sin aumentar el efecto antiinflamatorio ${ }^{(11)}$. La vía no genómica, a diferencia de la anterior, tiene un inicio de acción más rápido, un efecto antiinflamatorio más potente y se relaciona con menores efectos adversos, debido en parte a que su efecto es transitorio ${ }^{(41,42)}$. Esta vía se activa con dosis más altas de glucocorticoides, a partir de los $100 \mathrm{mg}$ de PDN o equivalente, alcanzando su máximo alrededor de los 250-500 mg, y es especialmente sensible a MTP y dexametasona.

En diversos estudios clínicos se ha demostrado que la activación preferencial de la vía no genómica mediante el uso de bolos intravenosos de MTP permite una rápida disminución de la prednisona oral asociado a menores dosis acumuladas y en consecuencia a menos efectos adversos a corto, mediano y largo plazo ${ }^{(43-45)}$. 


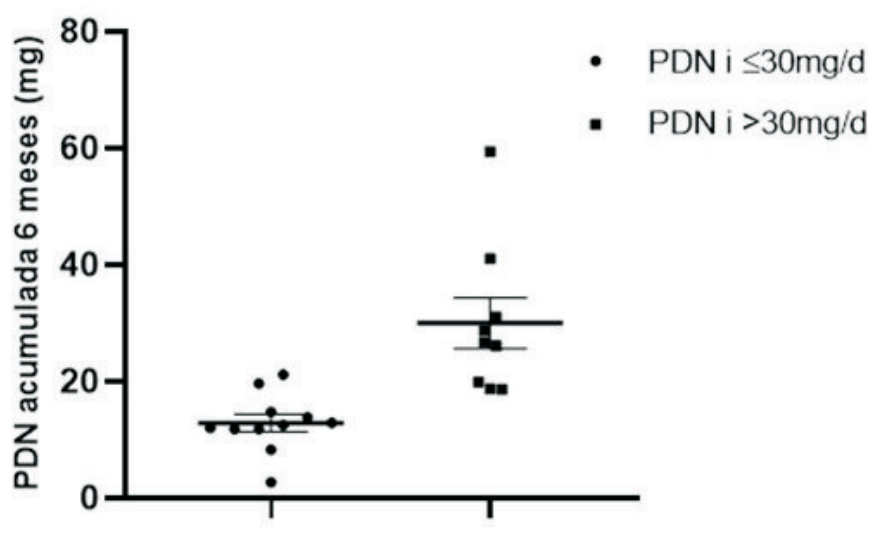

Figura 2. Comparación de la medida de dosis diaria acumulada de prednisona (PDN) en los primeros 6 meses de tratamiento entre los grupos según la dosis inicial. Se obtuvo una diferencia estadísticamente significativa entre los grupos PDN inicial $\leq 30 \mathrm{mg} / \mathrm{d}$ PDN inicial > $30 \mathrm{mg} / \mathrm{d}$; $\mathrm{p}=0,008 ; \mathrm{IC95 \%}$ : 8,20-26,13.

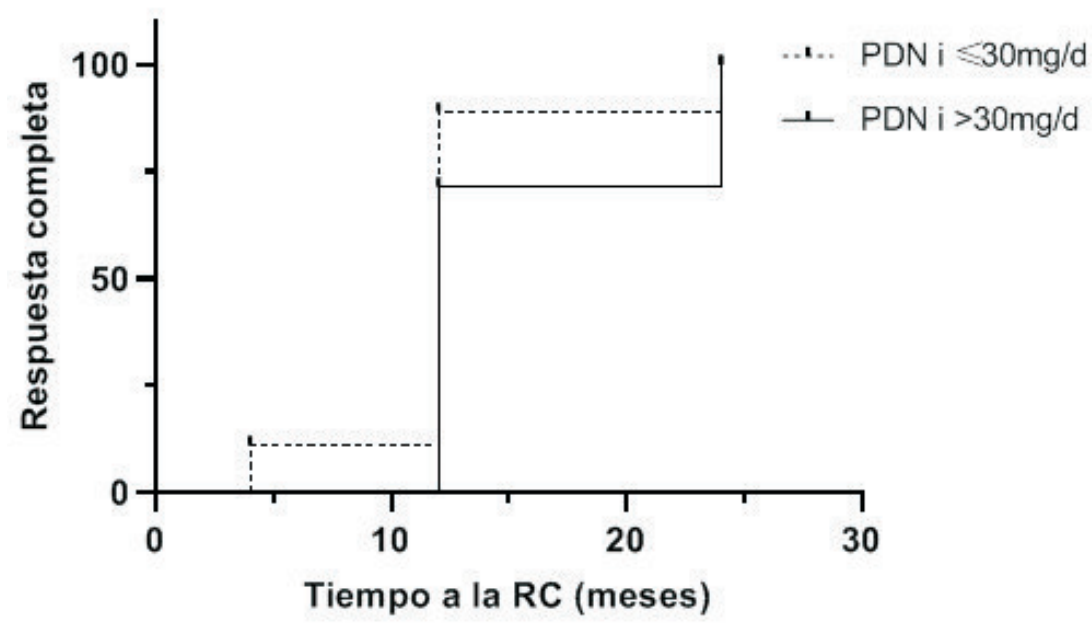

Figura 3. Curva de Kaplan Meier. Tiempo a la respuesta completa. No se observaron diferencias estadísticamente significativas en el tiempo a la respuesta completa entre ambos grupos. Se obtuvo un valor $p=0,27$ (test de log-rank).

Desde hace dos décadas, el estudio Euro Lupus Nephritis Trial (ELNT) incluyó bolos de MTP (tres pulsos intravenosos de $750 \mathrm{mg}$ cada uno) seguidos de dosis de prednisona $0,5 \mathrm{mg} / \mathrm{kg} / \mathrm{d}$, observando una eficacia comparable a dosis de prednisona más elevadas. En dicho trabajo aún persisten dosis acumuladas elevadas, en parte debido al lento descenso de prednisona, alcanzando $10 \mathrm{mg}$ a los 6 meses $^{(30)}$.

El estudio MYLUPUS es el único ensayo clínico aleatorizado y multicéntrico que comparó dosis medias de glucocorticoides orales $(0,5 \mathrm{mg} / \mathrm{kg} / \mathrm{d}, \mathrm{n}=39)$, con dosis altas $(1 \mathrm{mg} / \mathrm{kg} / \mathrm{d}, \mathrm{n}=42)$ durante la fase de inducción de la NL tipo III-IV asociadas a micofenolato. Las tasas de remisión completa en la semana 24 fueron similares para ambos grupos ( $19 \%$ vs. $21 \%$ y $67 \%$ vs. $56 \%$, respectivamente, valor $\mathrm{p}>0,05$ ) con menos infecciones en el grupo de dosis reducida de $\mathrm{PDN}^{(46)}$. En otro estudio observacional realizado por el grupo de investigación de Cruces-Bilbao, se compararon pacientes con NL clase III-IV-V de las cohortes Lupus-Cruces $(C C, n=29)$ y 
Lupus-Bordeaux ( $\mathrm{BC}, \mathrm{n}=44)$. El grupo $\mathrm{CC}$ presentó mayor número de pulsos de MTP (9,3 vs. 2,3, p <0,05), sin diferencia en la dosis acumulada de la misma. La proporción de pacientes con hidroxicloroquina $(100 \%$ vs $63 \%, \mathrm{p}<0,05)$ fue mayor en el CC. En relación con las dosis de prednisona oral, fueron menores para el grupo CC ( 21 frente a $42 \mathrm{mg} / \mathrm{d}, \mathrm{p}<0,05)$, así como también el número de semanas hasta $5 \mathrm{mg} / \mathrm{d}(12$ frente a $22, \mathrm{p}<0,05$ ) y las dosis medias diarias a los seis meses $(8,3$ frente a 21 $\mathrm{mg} / \mathrm{d}, \mathrm{p}<0,05)$. Las tasas de remisión renal completa fueron significativamente más altas en el CC a los seis $(69 \%$ frente a $30 \%, \mathrm{p}<0,05)$ y 12 meses $(86 \%$ frente a $43 \%$, p $<0,05)^{(45)}$. El análisis de regresión evidenció que el número de pulsos de MTP fue un predictor independiente para lograr la remisión completa y reducir los efectos adversos relacionados con los $\mathrm{GCC}^{(46)}$.

Más recientemente, el ensayo clínico AURA-LV, evaluó la eficacia y seguridad de agregar voclosporina, un inhibidor de la calcineurina, en dosis bajas y altas vs. placebo al tratamiento de inducción de la NL proliferativa asociado a MMF y glucocorticoides en dosis bajas. El diseño del estudio AURA-LV incluyó dosis de glucocorticoides más bajas en los tres grupos. Se usaron bolos de MTP intravenosa en dosis de hasta $250-500 \mathrm{mg}$ seguidos de PDN oral inicial hasta un máximo de 20-25 $\mathrm{mg} / \mathrm{d}$ y descenso rápido llegando a $2,5 \mathrm{mg} / \mathrm{d}$ a las $12 \mathrm{se}-$ manas. La eficacia del tratamiento en el grupo placebo respecto de otros estudios que emplean dosis más elevadas de glucocorticoides es similar ${ }^{(47)}$ e incluso se evidenciaron tasas de remisión más altas que en estudios anteriores $^{(46-48)}$.

Por último, el estudio Rituxilup, que consistió en la administración de rituximab y bolos intravenosos de MTP seguido de un tratamiento de mantenimiento con MMF sin glucocorticoides orales, dio como resultado la remisión completa en $72 \%$ de los pacientes con NL de clase III, IV o V en un período medio de 36 semanas ${ }^{(49)}$. Con esta evidencia, se ha llegado a plantear la discusión de si se puede prescindir del uso de glucocorticoides en el tratamiento de inducción de la $\mathrm{NL}^{(50)}$.

De acuerdo con la evidencia expuesta, cada vez contamos con más estudios que avalan que el uso de bolos intravenosos de MTP permite emplear dosis más bajas de prednisona. Con estos esquemas se logra igual tasa de remisión con menores efectos adversos y menor daño acumulado a largo plazo. Nuestros resultados están en línea con estas observaciones. En efecto, observamos mayor uso de pulsos intravenosos de MTP y menores dosis de PDN. Las guías actuales recomiendan el uso de dosis bajas de glucocorticoides con un rápido descenso de estos, para lo cual sugiere el uso de pulsos de $\mathrm{MTP}^{(16)}$.

Nuestro trabajo tiene limitaciones, derivadas de un análisis retrospectivo de un número de pacientes reduci- do y con un seguimiento de un año luego del inicio del tratamiento. Es llamativo el escaso uso de IECA, algo que deberá analizarse como un aspecto a corregir en el pautado de estos tratamientos. Por otro lado, la comparación de los efectos adversos fue escasa en la medida que el dato se recolectó de forma dicotómica, lo que puede determinar que exista un subregistro. Dentro de las fortalezas se destaca que por primera vez en nuestro medio se compara un esquema de dosis convencionales de PDN respecto a otro de dosis reducidas de PDN con pulsos de MTP. Asimismo, en nuestro medio son escasas las publicaciones sobre nefritis lúpica. En efecto, solemos utilizar evidencia extrapolada de estudios desarrollados en otros países, con otras características y otras poblaciones.

Este trabajo aporta nueva evidencia en nuestro medio respecto a que el tratamiento de la NL con dosis iniciales reducidas de prednisona, con descenso rápido, asociado al uso de pulsos intravenosos de MTP, más el empleo de inmunosupresores convencionales, en el contexto de un protocolo de tratamiento con uso sistemático de hidroxicloroquina, determina similares respuestas al esquema clásico de dosis iniciales de PDN altas. Asimismo, este esquema de tratamiento de la NL proliferativa que utilizamos se asoció a una menor dosis acumulada de prednisona lo que hace presumir una menor acumulación de daño relacionado con glucocorticoides a largo plazo.

\section{Abstract}

Introduction: current recommendations to treat lupus nephritis (LN) point to low-dose glucocorticoids to control the disease and avoid cumulativedamage.

Objective: to learn about and compare the response of patients with proliferative LN who are treated following two prednisone therapy guidelines: reduced initial doses $\leq 30 \mathrm{mg} / \mathrm{d}$ and standard initial doses $>30 \mathrm{mg} / \mathrm{d} \mathrm{du}-$ ring the induction stage.

Method: clinical, analytical and therapeutic guidelines of patients with proliferative $\mathrm{LN}$ were compared and classified into two groups according to the standard or low-dose initial prednisone dose.

Results: 21 patients with proliferative LN were studied ( $\mathrm{n}=12$ low-dose initial prednisonevs. $\mathrm{n}=9$ standard initial prednisone). No significant differences were found between clinical and analytical variables, although a significantly different statistic difference was observed in the number of methylprednisone pulses ( $5 \pm$ 2.95 initial prednisone $\leq 30 \mathrm{mg} / \mathrm{d}$ vs $2.33 \pm 2.91$ initial prednisone $>30 \mathrm{mg} / \mathrm{d}, \mathrm{p}=0.041$ ) and in the prednisone dose accumulated in 6 months $(12.8 \mathrm{mg} \pm 4.9$ initial prednisone $\leq 30 \mathrm{mg} / \mathrm{d}$ vs $30.0 \pm 13.1 \mathrm{mg}$ initial prednisone $>30 \mathrm{mg} / \mathrm{d}, \mathrm{p}=0.008$ ). 
No significant differences were seen between both groups in the proportion of patients who achieved complete response, neither in terms of the time it took to achieve it or in the side effects.

Conclusions: the treatment plan for the initial prednisone $\leq 30 \mathrm{mg} / \mathrm{d}$ was associated to a lower cumulative dose of response prednisone considering the comparable treatment, what suggests there being smaller cumulative harm as a consequence of the use of glucocorticoids.

\section{Resumo}

Introdução: as recomendações atuais para o tratamento da nefrite lúpica (NL) estão orientadas a doses de glicocorticoides mais baixas para controlar a enfermidade e evitar o dano acumulado.

Objetivo: conhecer e comparar a resposta ao tratamento de pacientes com NL proliferativa na etapa de indução com duas pautas de tratamento com prednisona (PDN): doses iniciais reduzidas $\leq 30 \mathrm{mg} / \mathrm{d}$ edoses iniciais padrão $>30 \mathrm{mg} / \mathrm{d}$.

Método: foram comparadas variáveis clínicas, analíticas e terapêuticas de pacientes com NL proliferativa divididos em dois grupos segundo a dose inicial de prednisona (PDNi) padrão ou reduzida.

Resultados: 21 pacientes com NL proliferativa $(\mathrm{n}=12$ PDNi reduzida vs. $n=9$ PDNi estândar) foram estudados. Não foram observadas diferenças significativas nas variáveis clínicas e analíticas. Observou-se uma diferença estatisticamente significativa no número de pulsos de metilprednisolona $(5 \pm 2,95 \mathrm{PDNi} \leq 30 \mathrm{mg} / \mathrm{d}$ vs $2,33 \pm 2,91$ PDNi $>30 \mathrm{mg} / \mathrm{d}, \mathrm{p}=0,041)$ e nas doses de prednisona acumulada aos 6 meses $(12,8 \mathrm{mg} \pm 4,9 \mathrm{PDNi}<30 \mathrm{mg} / \mathrm{d}$ vs 30,0 $\pm 13,1 \mathrm{mg}$ PDNi $>30 \mathrm{mg} / \mathrm{d}, \mathrm{p}=0,008)$. Não foram observadas diferenças significativas na proporção de pacientes que alcançaram a resposta completa, no tempo até alcançá-la nem nos efeitos adversos entre ambos grupos.

Conclusões: o esquema terapêutico do grupo PDNi $\leq 30 \mathrm{mg} / \mathrm{d}$ foi associado a uma menor dose acumulada de prednisona em resposta ao tratamento comparável, o que sugere menos dano cumulativo relacionado ao uso de glicocorticoides.

\section{Bibliografía}

1. Silvariño R, Ottati G, Noboa O. Nefropatía lúpica. Rev Méd Urug 2015; 31(1):64-78.

2. Cervera R, Khamashta M, Font J, Sebastiani G, Gil A, Lavilla $\mathbf{P}$, et al. Morbidity and mortality in systemic lupus erythematosus during a 10-year period: a comparison of early and late manifestations in a cohort of 1,000 patients. Medicine (Baltimore) 2003; 82(5):299-308.
3. Pons-Estel G, Catoggio L, Cardiel M, Bonfa E, Caeiro F, Sato E, et al. Lupus in Latin-American patients: lessons from the GLADEL cohort. Lupus 2015; 24(6):536-45.

4. Alarcón G. Cohortes multiétnicas de lupus: ¿qué nos han enseñado? Reumatol Clin 2011; 7(1):3-6. doi: 10.1016/j.reuma.2010.11.001.

5. Burgos P, McGwin GJr, Pons-Estel G, Reveille J, Alarcón G, Vilá L. US patients of Hispanic and African ancestry develop lupus nephritis early in the disease course: data from LUMINA, a multiethnic US cohort (LUMINA LXXIV). Ann Rheum Dis 2011; 70(2):393-4.

6. Contreras G, Lenz O, Pardo V, Borja E, Cely C, Iqbal K, et al. Outcomes in African Americans and Hispanics with lupus nephritis. Kidney Int 2006; 69(10):1846-51.

7. Garau M, Cabrera J. Informe del Registro Uruguayo de Glomerulopatías 2018: datos de los años 2016-2017. Montevideo: Programa de Prevención y Tratamiento de las Glomerulopatías, 2018. Disponible en: https://www.nefrologia.hc.edu.uy/images/RUG_Informe2018_1.pdf. [Consulta: 12 abril 2021].

8. Garau M, Cabrera J, Ottati G, Caorsi H, González Martínez F, Acosta N, et al. Temporal trends in biopsy proven glomerular disease in Uruguay, 1990-2014. PLoS One 2018; 13(10):e0206637. doi: 10.1371/journal.pone.0206637.

9. Urowitz M, Gladman D, Tom B, Ibañez D, Farewell V. Changing patterns in mortality and disease outcomes for patients with systemic lupus erythematosus. J Rheumatol 2008; 35(11):2152-8.

10. Thamer M, Hernán M, Zhang Y, Cotter D, Petri M. Prednisone, lupus activity, and permanent organ damage. J Rheumatol 2009; 36(3):560-4.

11. Ruiz-Irastorza G, Danza A, Khamashta M. Glucocorticoid use and abuse in SLE. Rheumatology (Oxford) 2012; 51(7):1145-53.

12. Al Sawah S, Zhang X, Zhu B, Magder L, Foster S, Iikuni $\mathbf{N}$, et al. Effect of corticosteroid use by dose on the risk of developing organ damage over time in systemic lupus erythematosus-the Hopkins Lupus Cohort. Lupus Sci Med 2015; 2(1):e000066. doi: 10.1136/lupus-2014-000066.

13. Apostolopoulos D, Morand E. It hasn't gone away: the problem of glucocorticoid use in lupus remains. Rheumatology (Oxford) 2017; 56(Suppl 1):i114-22. doi: 10.1093/rheumato$\log /$ kew406.

14. Tani C, Vagelli R, Stagnaro C, Carli L, Mosca M. Remission and low disease activity in systemic lupus erythematosus: an achievable goal even with fewer steroids? Real-life data from a monocentric cohort. Lupus Sci Med 2018; 5(1):e000234. doi: 10.1136/lupus-2017-000234.

15. Petri M, Magder L. Comparison of remission and lupus low disease activity state in damage prevention in a united states systemic lupus erythematosus cohort. Arthritis Rheumatol 2018; 70(11):1790-5.

16. Fanouriakis A, Kostopoulou M, Cheema K, Anders H, Aringer M, Bajema I, et al. 2019 Update of the Joint European League against rheumatism and European Renal Asso- 
ciation-European Dialysis and Transplant Association (EULAR/ERA-EDTA) recommendations for the management of lupus nephritis. Ann Rheum Dis 2020; 79(6):713-23.

17. Ruiz-Arruza I, Ugarte A, Cabezas-Rodríguez I, Medina J, Moran M, Ruiz-Irastorza G. Glucocorticoids and irreversible damage in patients with systemic lupus erythematosus. Rheumatology (Oxford) 2014; 53(8):1470-6.

18. Danza A, Narváez J, Graña D, Pérez L, Viera A, Baccelli A, et al. Relación entre el uso de glucocorticoides y el daño crónico en Lupus Eritematoso Sistémico: una asociación precoz y nociva. Estudio exploratorio. Rev Urug Med Int 2021; 6(1):14-23.

19. Ruiz-Irastorza G, García M, Espinosa G, Caminal L, Mitjavila F, González-León R, et al. First month prednisone dose predicts prednisone burden during the following 11 months: an observational study from the RELES cohort. Lupus Sci Med 2016; 3(1):e000153. doi: 10.1136/lupus-2016-000153.

20. Dixon W, Suissa S, Hudson M. The association between systemic glucocorticoid therapy and the risk of infection in patients with rheumatoid arthritis: systematic review and meta-analyses. Arthritis Res Ther 2011; 13(4):R139. doi: $10.1186 /$ ar3453.

21. George M, Baker J, Winthrop K, Hsu J, Wu Q, Chen L, et al. Risk for serious infection with low-dose glucocorticoids in patients with rheumatoid arthritis: a cohort study. Ann Intern Med 2020; 173(11):870-8.

22. Youssef J, Novosad S, Winthrop K. Infection risk and safety of corticosteroid use. Rheum Dis Clin North Am 2016; 42(1):157-76. doi: 10.1016/j.rdc.2015.08.004.

23. Jick S, Lieberman E, Rahman M, Choi H. Glucocorticoid use, other associated factors, and the risk of tuberculosis. Arthritis Rheum 2006; 55(1):19-26.

24. World Health Organization. Latent tuberculosis infection: updated and consolidated guidelines for programmatic management. Geneva: WHO, 2018. Disponible en: https://www.ncbi.nlm.nih.gov/books/NBK531235/pdf/Book shelf_NBK531235.pdf. [Consulta: 26 setiembre 2020].

25. Danza A, Ruiz-Irastorza G. Infection risk in systemic lupus erythematosus patients: susceptibility factors and preventive strategies. Lupus 2013; 22(12):1286-94.

26. Ruiz-Irastorza G, Ugarte A, Saint-Pastou Terrier C, Lazaro E, Iza A, Couzi L, et al. Repeated pulses of methyl-prednisolone with reduced doses of prednisone improve the outcome of class III, IV and V lupus nephritis: An observational comparative study of the Lupus-Cruces and lupus-Bordeaux cohorts. Autoimmun Rev 2017; 16(8):826-32.

27. Hochberg MC. Updating the American College of Rheumatology revised criteria for the classification of systemic lupus erythematosus. Arthritis Rheum 1997; 40(9):1725. doi: 10.1002/art.1780400928.

28. Weening J, D'Agati V, Schwartz M, Seshan S, Alpers C, Appel G, et al. The classification of glomerulonephritis in systemic lupus erythematosus revisited. J Am Soc Nephrol
2004; 15(2):241-50. doi: 10.1097/01.asn.0000108969 $21691.5 \mathrm{~d}$.

29. Montañés Bermúdez R, Bover Sanjuán J, Oliver Samper A, Ballarín Castán J, Gràcia García S. Valoración de la nueva ecuación CKD-EPI para la estimación del filtrado glomerular. Nefrología (Madr.) 2010; 30(2):185-94.

30. Houssiau F, Vasconcelos C, D'Cruz D, Sebastiani G, Garrido Ed R, Danieli M, et al. Immunosuppressive therapy in lupus nephritis: the Euro-Lupus Nephritis Trial, a randomized trial of low-dose versus high-dose intravenous cyclophosphamide. Arthritis Rheum 2002; 46(8):2121-31.

31. Gladman D, Ibañez D, Urowitz M. Systemic lupus erythematosus disease activity index 2000. J Rheumatol 2002; 29(2):288-91.

32. Osakidetza. Servicio Vasco de Salud. Hospital Universitario Cruces. Servicio de Medicina Interna, Unidad de Enfermedades Autoinmunes. Autoinmunes. Mobile Application Version 1.05. Osakidetza: Hospital Universitario Cruces, 2019. Disponible en: https://play.google.com/store/apps/details?id=com.ionicframework.apautoinmunes2327096\&hl=es\&gl=US. [Consulta: 15 setiembre 2020].

33. Tarr T, Papp G, Nagy N, Cserép E, Zeher M. Chronic high-dose glucocorticoid therapy triggers the development of chronic organ damage and worsens disease outcome in systemic lupus erythematosus. Clin Rheumatol 2017; 36(2):327-33.

34. Ruiz-Irastorza G, Danza A, Khamashta M. Tratamiento del lupus eritematoso sistémico: mitos, certezas y dudas. Med Clin (Barc) 2013; 141(12):533-42.

35. Little J, Parker B, Lunt M, Hanly J, Urowitz M, Clarke A, et al. Glucocorticoid use and factors associated with variability in this use in the Systemic Lupus International Collaborating Clinics Inception Cohort. Rheumatology (Oxford) 2018; 57(4):677-87.

36. Ruiz-Arruza I, Lozano J, Cabezas-Rodríguez I, Medina J, Ugarte A, Erdozain J, et al. Restrictive use of oral glucocorticoids in systemic lupus erythematosus and prevention of damage without worsening long-term disease control: an observational study. Arthritis Care Res (Hoboken) 2018; 70(4):582-91.

37. Petri M, Purvey S, Fang H, Magder L. Predictors of organ damage in systemic lupus erythematosus: the Hopkins Lupus Cohort. Arthritis Rheum 2012; 64(12):4021-8.

38. Cassano G, Roverano S, Paira S, Bellomio V, Lucero E, Berman A, et al. Accrual of organ damage over time in Argentine patients with systemic lupus erythematosus: a multi-centre study. Clin Rheumatol 2007; 26(12):2017-22.

39. Buttgereit F, Wehling M, Burmester G. A new hypothesis of modular glucocorticoid actions: steroid treatment of rheumatic diseases revisited. Arthritis Rheum 1998; 41(5):761-7.

40. Mejía-Vilet J, Ayoub I. The use of glucocorticoids in lupus nephritis: new pathways for an old drug. Front Med (Lausanne) $2021 ; 8: 622225$. doi: 10.3389/fmed.2021.622225

41. Buttgereit F, Straub R, Wehling M, Burmester G. Glucocorticoids in the treatment of rheumatic diseases: an update 
on the mechanisms of action. Arthritis Rheum 2004; 50(11):3408-17.

42. Panettieri R, Schaafsma D, Amrani Y, Koziol-White C, Ostrom R, Tliba O. Non-genomic effects of glucocorticoids: an updated view. Trends Pharmacol Sci 2019; 40(1):38-49.

43. Badsha H, Edwards C. Intravenous pulses of methylprednisolone for systemic lupus erythematosus. Semin Arthritis Rheum 2003; 32(6):370-7.

44. Parker B, Bruce I. High dose methylprednisolone therapy for the treatment of severe systemic lupus erythematosus. Lupus 2007; 16(6):387-93.

45. Ruiz-Irastorza G, Ruiz-Estevez B, Lazaro E, Ruiz-Arruza I, Duffau P, Martin-Cascon M, et al. Prolonged remission in SLE is possible by using reduced doses of prednisone: An observational study from the Lupus-Cruces and Lupus-Bordeaux inception cohorts. Autoimmun Rev 2019; 18(9):102359. doi: 10.1016/j.autrev.2019.102359.

46. Zeher M, Doria A, Lan J, Aroca G, Jayne D, Boletis I, et al. Efficacy and safety of enteric-coated mycophenolate sodium in combination with two glucocorticoid regimens for the treatment of active lupus nephritis. Lupus 2011; 20(14):1484-93.

47. Rovin B, Solomons N, Pendergraft W3rd, Dooley M, Tumlin J, Romero-Diaz J, et al. A randomized, controlled double-blind study comparing the efficacy and safety of dose-ranging voclosporin with placebo in achieving remission in patients with active lupus nephritis. Kidney Int 2019; 95(1):219-31.

48. Sinclair A, Appel G, Dooley M, Ginzler E, Isenberg D, Jayne D, et al. Mycophenolate mofetil as induction and maintenance therapy for lupus nephritis: rationale and protocol for the randomized, controlled Aspreva Lupus Management Study (ALMS). Lupus 2007; 16(12):972-80.

49. Condon M, Ashby D, Pepper R, Cook H, Levy J, Griffith $\mathbf{M}$, et al. Prospective observational single-centre cohort study to evaluate the effectiveness of treating lupus nephritis with rituximab and mycophenolate mofetil but no oral steroids. Ann Rheum Dis 2013; 72(8):1280-6.

50. Lightstone L, Doria A, Wilson H, Ward F, Larosa M, Bargman J. Can we manage lupus nephritis without chronic corticosteroids administration? Autoimmun Rev 2018; 17(1):4-10. doi: 10.1016/j.autrev.2017.11.002 .

\section{Contribución de autores}

Adriana Carlomagno, ORCID 0000-0001-7350-366X. Concepción, diseño, ejecución, análisis, interpretación de los resultados, redacción, revisión crítica.

Gonzalo Silveira, ORCID 0000-0002-5582-4563. Concepción, diseño, ejecución, análisis, interpretación de los resultados, redacción, revisión crítica.

Carina Pizzarrosa, ORCID 0000-0001-5449-9124. Concepción, diseño, ejecución, análisis, interpretación de los resultados, revisión crítica. Álvaro Danza, ORCID 0000-0001-9070-2230. Concepción, diseño, análisis, interpretación de los resultados, redacción, revisión crítica. Martín Rebella, ORCID 0000-0002-1925-4022. Concepción, diseño, análisis, interpretación de los resultados, redacción, revisión crítica. Martín Yandián, ORCID 0000-0001-9446-4778. Interpretación de los resultados, revisión crítica.

Federico Yandián, ORCID 0000-0001-8374-9508. Interpretación de los resultados, revisión crítica. 